\title{
A Mutli-Agent Task Allocation Model in Open-Space Emergency Evacuation Management
}

\author{
Lei Du*, Guixing Zheng, Yuan Kan, Bo Xu and Jianhua Kang \\ Department of Basic Courses, \\ Academy of Military Transportation, PLA, Tianjin, China \\ *dulei001@126.com
}

\begin{abstract}
Open-space emergency evacuation is the movement of people away the threat in the short time. Task allocation is a fundamental research challenge in emergency evacuation domain of multi-agent systems (MAS). This paper proposes the multi-agent task allocation model which is called EV-GAP. Accordingly, the capability of agent was redefined to satisfy the requirement of open-space emergency evacuation. Corresponding the task allocation algorithm based on EV-GAP has is also given. Finally, the experiment of the task allocation of open-space emergency evacuation for a chlorine leakage accident is completed to validate this model.
\end{abstract}

Keywords: emergency evacuation, multi-agent systems, task allocation, EV-GAP

\section{Introduction}

Currently, public emergency event occurs frequently in the world. As an important measure, emergency evacuation can be adopted to deal with a variety of public emergency events, such as floods, hurricanes, earthquakes, chemical spill, fire, and so on. Therefore, emergency evacuation has become a hotspot of research.

In the past ten years, the issue of emergency evacuation within the enclosed space (such as large buildings, boats, aircraft, etc.) has been generally researched. On the other hand, the study of open-space emergency evacuation was also emphasized recently. For open-space emergency evacuation management, its core issue is how to allocate the emergency evacuation tasks to agents rapidly and effectively to minimize the loss of the affected population.

In essence, task is a specific description of solution for any question in accordance with its own characteristics. The problem of task allocation first appeared in the various types of production, planning and flexible manufacturing systems. And it is a typical combinatorial optimization problem. The traditional task allocation problem is to decompose a problem into multiple sub-tasks, and assume that all processors can handle the same tasks without discrimination. This task allocation problem have wide applications in many fields, such as vehicle scheduling, assignment problem, a multi-processor parallel processing, grid computing, etc.

The multi-agent system is a loosely coupled system which consists of a number of interactive agent calculation units. In multi-agent systems, task allocation problem is how to choose the right agent to complete various tasks. Gerkey [1] gave an analysis of multi-robot task allocation and coordination mechanism. Paper [2] proposed a distributed parallel multi- 
task allocation algorithm among self-organizing and self-learning agents. And paper [3] discussed the clustering-based coalition formation and self-adjustment mechanisms for tasks in the wireless sensor network. Moreover, paper [4] designed a mathematical model for balanced multi-robot task allocation problem based on K-means clustering and auction based mechanisms.

For multi-robot system, paper [5] researched the task assignment of agent teams in RoboCupRescue project. In paper [6], the reinforcement learning algorithm for multi-robot is divided into two types: non-cooperation and cooperation. Then, this paper proposed multirobot task allocation algorithm satisfies the requirement of dynamic task allocation for firedisaster response. And paper [7] presented task allocation algorithms for a multi-robot system where the tasks are divided into disjoint groups and there are precedence constraints between the task groups. Paper [8] proposed a modified particle swarm optimization (PSO) algorithm to deal with the allocation problem of earthquake emergency shelter.

In emergency management domain, paper [9] constructed the Markov Decision Processes(MDP) model for dynamic emergency task allocation under resource constraints. Jones [10] proposed a market-based task allocation system for disaster response. Moreover, paper [11] presents an open multi-agent system, called Overseer, which leverages context information in a mobile setting to facilitate collaboration and task allocation for disaster response. For distributing rescue agents, paper [12] provided an approach in a more rational way, by using combinatorial auction techniques to perform task allocation.

In recent years, a variety of practical research of multi-agent task al-location has been developed $[13,14,15]$. But the various models are usually for a specific field or general field, the specific impact of the open-space emergency evacuation has not been considered. Consequently, this paper proposed a multi-agent task allocation model, called EV-GAP, to resolve this problem in open-space emergency evacuation management.

\section{Task Allocation Model for Open-Space Emergency Evacuation}

\subsection{GAP}

In open-space emergency evacuation management domain, how to assign tasks to agents under dynamic conditions is a very important problem. And the Generalized Assignment Problem (GAP) is also known to be NP-complete [16]. In order to maximize the total reward of open-space emergency evacuation system, GAP can be formalized as follows.

1. The set of tasks can defined as $J$, and the set of agents can be defined as $I$. The set of tasks need to be allocated to the set of agents.

2. Any agent $i \in I$ is made within the restraints of emergency resources.

3. The task $j \in J$ will consumes $c_{i j}$ unit of emergency resources when the task $j$ is executed by agent $i$.

4. Agent $i$ has the capability $k_{i j}\left(0 \leq k_{i j} \leq 1\right)$ to carry out the task $j$.

5. There is the matrix $A$ to be described the task allocation. The formula $a_{i j}=1$ can be represented that task $j$ is allocated to the agent $i$. And the formula $a_{i j}=0$ is true, when task $j$ is not allocated to the agent $i$ 
An optimum task allocation is given by matrix $A^{*}$ to maximize the emergency evacuation system reward. One task will be assigned to only one agent. And the constraint of emergency resource can be considered. The matrix $A^{*}$ can be defined as follows.

$$
\begin{aligned}
& A^{*}=\arg \max _{A^{\prime}} \sum_{i \in I} \sum_{j \in J} k_{i j} \times a_{i j}^{\prime} \\
& \forall i \in I, \sum_{j \in J} c_{i j} \times a_{i j} \leq r_{i} \\
& \forall j \in J, \sum_{i \in I} a_{i j} \leq 1
\end{aligned}
$$

\subsection{E-GAP}

Paper [17] proposed the extended-GAP(E-GAP) which considered the element of time. So the dynamic tasks can be described exactly by using E-GAP. The total reward $W$ can be computed in E-GAP as the sum of the agents' partial rewards over time. The variable $d_{j}^{t}$ is the expense of any emergency evacuation task which is not be allocated to agent. So the task allocation can be defined as follows.

$$
\begin{aligned}
& W=\sum_{t} \sum_{i^{t} \in I^{t}} \sum_{j^{t} \in J^{t}} w_{i j}^{t} \times a_{i j}^{t}-\sum_{t} \sum_{j^{t} \in J^{t}}\left(1-\sum_{i^{t} \in I^{t}} a_{i j}^{t}\right) \times d_{j}^{t} \\
& \forall t \forall i^{t} \in I^{t}, \sum_{j \in J^{t}} c_{i j}^{t} \times a_{i j}^{t} \leq r_{i}^{t} \\
& \forall t \forall j^{t} \in J^{t}, \sum_{i \in I^{t}} a_{i j}^{t} \leq 1
\end{aligned}
$$

And it was considered that AND restraints have been existed in the tasks. The task set $\alpha_{k}=\left\{j_{k_{1}}, \cdots, j_{k_{q}}\right\}$ can be grouped by AND restraints. And the task group set can be defined as $\theta=\left\{\alpha_{1}, \cdots, \alpha_{p}\right\}$. Thus, the partial reward $w_{i j}$ for allocating task $j$ to agent $i$ is given by Eq.7.

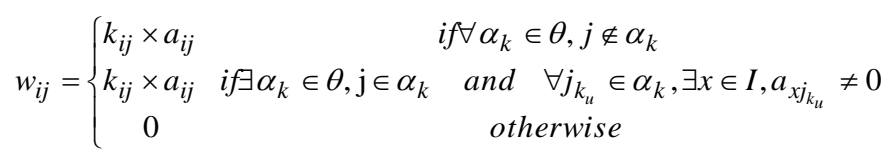

\section{The Multi-Agent Task Allocation Model EV-GAP}

\subsection{The Capability of Emergency Evacuation Agent}

According to the E-GAP Model, the agent's capability for the allocated task is the most important element in the open-space emergency evacuation management. We defined the capability of agent in order to represent the reward of evacuation system accurately. So the agent's capability for the task can be defined as follows.

$$
k_{i j}^{t}=y_{i j}^{t} \times \alpha+s_{i j}^{t} \times(1-\alpha)
$$

The agent's capability $k_{i j}^{t}$ depends on the significance of task $j$ and the distance between the position of agent $i$ and task $j$. In Eq.8, the variable $y_{i j}^{t}$ represents the significance of 
emergency evacuation task $j$ by agent $i$ at time $t$. The variable $s_{i j}^{t}$ is the parameter of distance between agent $i$ and task $j$. And the variable $\alpha \leq 1$ is the weight coefficient.

\subsubsection{The Importance of Emergency Evacuation Task}

The variable $y_{i j}^{t}$ is the importance of emergency evacuation task. And one of the determinants is variable $d_{i j}^{t}$ which is the distance between the sources of danger and the place of the task at time $t$. Another is the number of population distribution near the place of the task $j$ at time $t$. Therefore, variable $y_{i j}^{t}$ can be defined as follows.

$$
y_{i j}^{t}=d_{i j}^{t}+p_{i j}^{t}
$$

The purpose of emergency evacuation is the movement of the people from disaster areas to safe areas. However, usually the sources of danger would threaten the safety of the people of the surrounding area. And most of dangerous source will be spread with the development of the incident, such as tsunami, nuclear leaks, volcanic eruptions. Because emergency evacuation is to be completed within the time limit, the priority of the emergency task will be also affected by the distance between the tasks and dangerous source.

To consider multiple sources of danger in the presence of sources of danger set $U$, the distance parameter between the task $j$ and dangerous source can be calculated as follows.

$$
d_{i j}^{t}=\frac{\max _{n \in J, m \in U}\left\{c^{t}(n, m)\right\}-\min _{m \in U}\left\{c^{t}(j, m)\right\}}{\max _{n \in J, m \in U}\left\{c^{t}(n, m)\right\}}
$$

In Eq.10, the Euclidean distance has been adopted because that transport network is not affected by the development of emergency event. So the variable $c^{t}(n, m)$ was described as the Euclidean distance between the task $j$ and dangerous source. And $\max _{n \in J, m \in U}\left\{c^{t}(n, m)\right\}$ is the maximum distance, $\min _{m \in U}\left\{c^{t}(j, m)\right\}$ is the minimal one.

The variable $p_{i j}^{t}$ is the parameter of the population distribution number near the place of task $j$ at time $t$. It can be calculated as follows.

$$
p_{i j}^{t}=q_{i j}^{t} \times \gamma
$$

In Eq.11, the variable $q_{i j}^{t}$ is the number of population. And the weight coefficient $\gamma$ can be determined by the importance of task $j$ for the people near the place and the personnel organization, such as age, occupation, etc.

In this paper, the people number is estimated by analysis of the erlang of mobile telephone base station near the dangerous source. An erlang is a dimensionless unit that is used in telephony as a statistical measure of offered load or carried load on service-providing elements such as telephone circuits or telephone switching equipment. Erlang $E$ which is related to the call arrival rate $\lambda$ and the average call-holding time ${ }^{h}$ can be defined as Eq.12.

$$
E=\lambda \times h
$$


Thus, the people number $q_{i j}^{t}$ is given by Equation 13. And the variable $\omega_{k}$ is the gain coefficient.

$$
q_{i j}^{t}=\Sigma \frac{E_{k}}{h_{k}} \times \omega_{k}
$$

\subsubsection{The Distance Parameter}

The transport cost of emergency agent can be expressed in terms of the distance between agent $i$ and task $j$. So the distance parameter $s_{i j}^{t}$ can be defined as follows.

$$
s_{i j}^{t}=\frac{\max _{n \in J}\left\{D^{t}(i, n)\right\}-D^{t}(i, j)}{\max _{n \in J}\left\{D^{t}(i, n)\right\}} \times \delta
$$

In Eq.14, $\max _{n \in J}\left\{D^{t}(i, n)\right\}$ is the maximum distance between agent $i$ and tasks. And the distance between agent $i$ and task $j$ is represented as the variable $D^{t}(i, j)$. The implementation capacity of agent $i$ is reduced with increasing distance from the task $j$.

In order to accurately describe the cost of agent arrives to the location to perform task, the real-time speed variable $v$ was been introduced from external sensors. And the invariant $V$ is the set speed value. The speed coefficient $\delta$ can be calculated as follows.

$$
\delta= \begin{cases}1 & v \geq V \\ \frac{v}{V} & v<V\end{cases}
$$

\subsection{Task Allocation Algorithm based on EV-GAP}

We redefined the emergency response capacity of agent in open-space emergency evacuation management. The multi-agent task allocation algorithm based on the agent's capacity can be proposed as follows.

Input: task set $\mathrm{J}$, agent set $\mathrm{I}$, resource set $\mathrm{R}$, dangerous source set $\mathrm{U}$, task allocation set A

Output: task allocation plan $\mathrm{g}$

1. for all $\mathrm{a} \in \mathrm{A}$ do $/ /$ loop of task allocation matrix $\mathrm{a} \in \mathrm{A}$

2. $\quad \operatorname{wmax}:=0$;

3. $\mathrm{w}:=0$;

4. for all $\mathrm{i}, \mathrm{j} \in \mathrm{a}$ do $/ /$ the calculate process of maximal reward of the matrix a

5. $y:=\operatorname{Dan}(\mathrm{j}, \mathrm{J}, \mathrm{U}) ; / /$ the importance of task was to be calculated on the basis of Eq.9-13

6. $s:=\operatorname{Dist}(\mathrm{i}, \mathrm{j}, \mathrm{J}) ; \quad / /$ the distance parameter was to be calculated on the basis of Eq.14-15

7. $\mathrm{kij}:=\operatorname{cap}(\mathrm{y}, \mathrm{b}, \mathrm{s}) ; / /$ the capability of agent was to be calculated on the basis of Eq.8

8. w:=w+kij $\times a i j$;

9. end-for

10. if $w \max <\mathrm{w}$ then // make the matrix a as task allocation plan based on the reward $\mathrm{w}$ 

11. $\quad w \max :=\mathrm{w}$;
12. $\mathrm{g}:=\mathrm{a}$;
13. end-if
14. end-for
15. return $\mathrm{g} \quad / /$ output the task allocation plan $\mathrm{g}$

\section{Experiments and Results}

The multi-agent task allocation model can be used to provide the decision support information for the emergency evacuation decision-maker. According to the segment of a chlorine leakage accident, we verify the validity of the EV-GAP model in this paper.

A chlorine leakage accident process can be described simply. Chlorine leakage accident occurred in an area. Emergency decision-maker decides to evacuate 782 people away the place of chlorine leakage accident by 10 bus evacuation vehicles, shown in Figure 1.

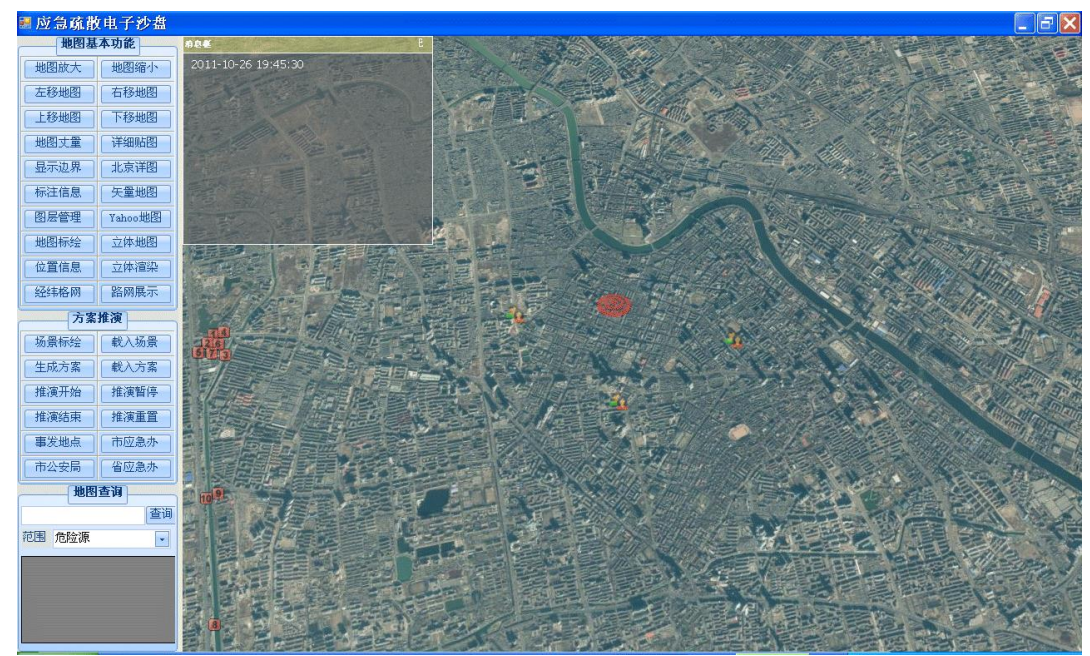

\section{Figure 1. Emergency Evacuation State for Chlorine Leakage Accident}

We introduce the real-time speed data of vehicles from external sensor of department of transport in order to represent the real scene. According to the real-time speed data, the evacuation area can be described by different colors as Figure 2. The red line indicates the speed below $20 \mathrm{~km} / \mathrm{h}$. The pink line indicates the speed between $20 \mathrm{~km} / \mathrm{h}$ and $40 \mathrm{~km} / \mathrm{h}$. The orange line indicates the speed between $40 \mathrm{~km} / \mathrm{h}$ and $60 \mathrm{~km} / \mathrm{h}$. Finally, the green line indicates the speed more than $60 \mathrm{~km} / \mathrm{h}$. 


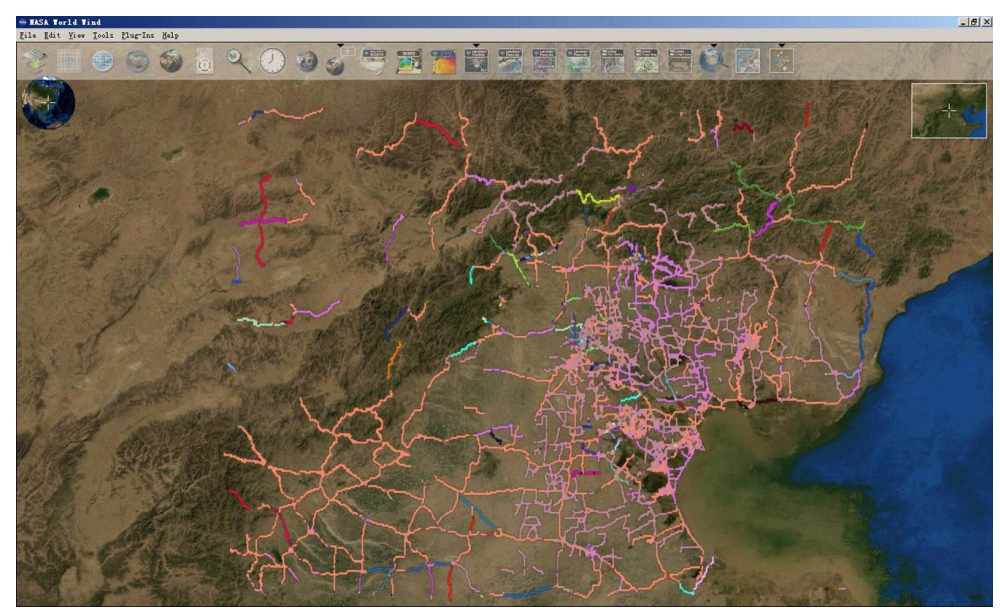

Figure 2. Real-time Speeds of Vehicles

In this scene, the vehicles are the agents. And the emergency tasks are the movement of people near dangerous source. The task allocation matrix set $A$ can be simplified as follows:

$$
\begin{aligned}
& A=\left\{A_{1}, A_{2}, A_{3}\right\}, \\
& \begin{array}{llllllllll}
i_{1} & i_{2} & i_{3} & i_{4} & i_{5} & i_{6} & i_{7} & i_{8} & i_{9} & i_{10}
\end{array} \\
& A_{1}=\begin{array}{lllllllllll}
j_{1} & 1 & 1 & 0 & 0 & 0 & 0 & 0 & 1 & 0 & 0 \\
j_{2} & 0 & 0 & 1 & 1 & 1 & 1 & 0 & 0 & 1 & 0
\end{array} \\
& \begin{array}{lllllllllll}
j_{3} & 0 & 0 & 0 & 0 & 0 & 0 & 1 & 0 & 0 & 1
\end{array} \\
& \begin{array}{llllllllll}
i_{1} & i_{2} & i_{3} & i_{4} & i_{5} & i_{6} & i_{7} & i_{8} & i_{9} & i_{10}
\end{array} \\
& A_{3}=\begin{array}{lllllllllll}
j_{1} & 1 & 1 & 1 & 0 & 0 & 0 & 0 & 0 & 0 & 0 \\
j_{2} & 0 & 0 & 0 & 1 & 1 & 1 & 1 & 0 & 1 & 0
\end{array} \\
& \begin{array}{lllllllllll}
j_{3} & 0 & 0 & 0 & 0 & 0 & 0 & 0 & 1 & 0 & 1
\end{array}
\end{aligned}
$$

The plan $A_{1}$ is the best one which can be get maximum rewards from the chlorine leakage accident evacuation though the emergency evacuation task allocation algorithm based on the EV-GAP. And we make an experiment with the task allocation plans includes $A_{1}, A_{2}, A_{3}$ for the chlorine leakage accident by MATSim(Multi-Agent Transport Simulation Toolkit) [18][19] at AM8:00, PM16:00 and PM24:00 in one day. The simulation results are shown in Figure 3, Figure 4 and Figure 5. It is well known that time is crucial to the emergency evacuation. So the plan $A_{1}$ is the best one because that the emergency evacuation time of plan $A_{1}$ is shortest in three simulations. The effectiveness of the emergency evacuation task allocation model EV-GAP can be proved with this experiment. 
International Journal of Hybrid Information Technology Vol.6, No.5 (2013)

emergency evacuation process

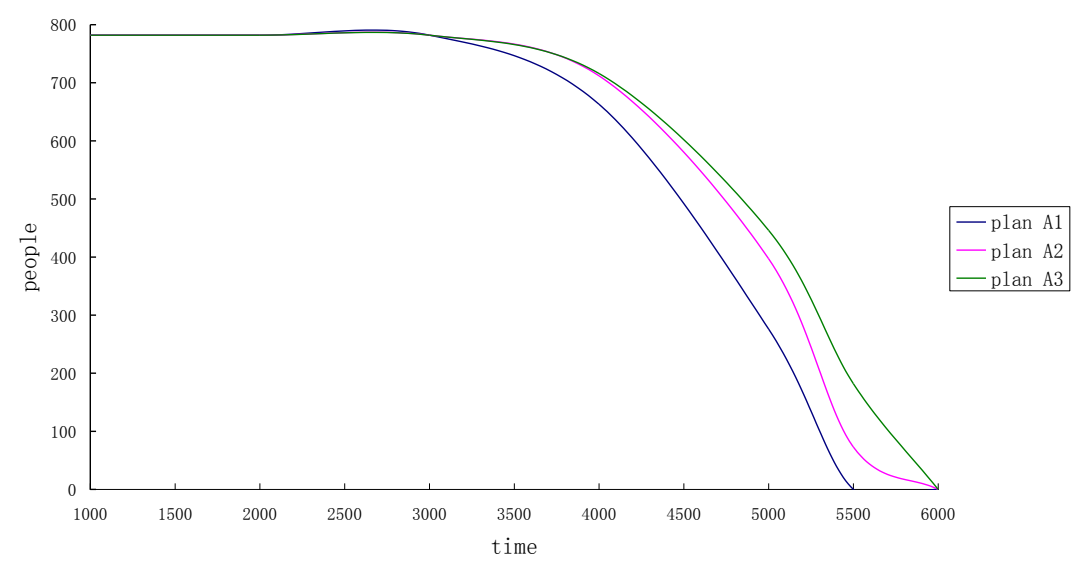

Figure 3. Emergency evacuation at AM 8:00

emergency evacuation process

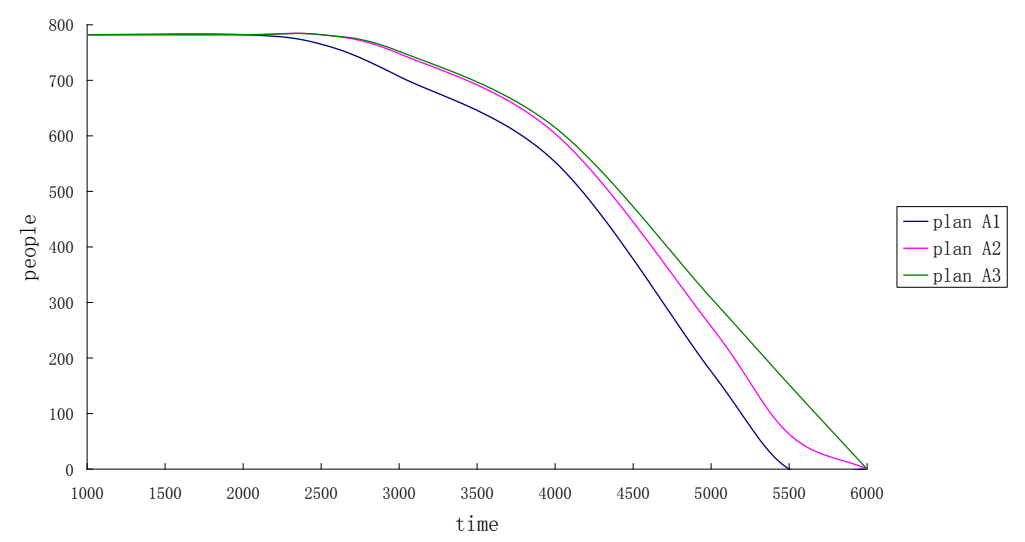

Figure 4. emergency evacuation at PM 16:00

emergency evacuation process

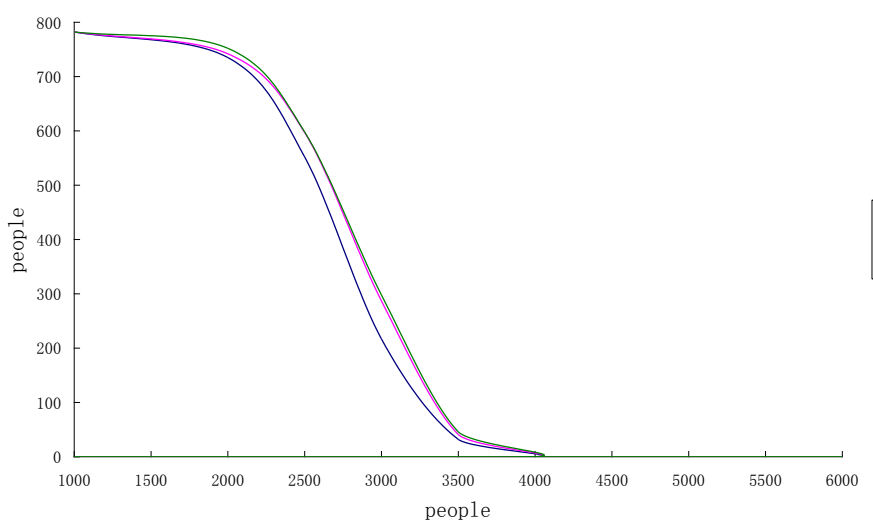

Figure 5. emergency evacuation at PM 24:00 


\section{Conclusions}

In this paper, the multi-agent task allocation model in open-space emergency evacuation management has been established. We propose the emergency evacuation task allocation model EV-GAP, and redefined the capability of agent. Then, the task allocation algorithm is also given. Finally, the effectiveness of the EV-GAP model has been proved though the experiment.

In future work, we are thinking of incorporating the individual intention to the task allocation model, and addressing issues such as team decision-making in open-space emergency evacuation management.

\section{Acknowledgements}

This paper is support by science and technology support key project plan of Tianjin (10ZCGYGX18600) and Small and medium-sized enterprises technology innovation fund of Tianjin (11ZXCXGX07700).

\section{References}

[1] B. P. Gerkey and M. J. Mataric, "A formal analysis and taxonomy of task allocation in multi-robot systems", Robotics Research, vol. 23, no. 9, (2004), pp. 939-954.

[2] Z. -P. Su, J. -G. Jiang, C. -Y. Liang and G. -F. Zhang, "A Distributed Algorithm for Parallel Multi-task Allocation Based on Profit Sharing Learning”, Acta Automatica Sinica, vol. 37, no. 7, (2011), pp. 865-872.

[3] Y. Yang, X. Qiu, L. Meng and K. Long, "Task coalition formation and self-adjustment in the wireless sensor networks", International Journal of Communication Systems, (2012).

[4] M. Elango, S. Nachiappan and M. K. Tiwari, "Balancing task allocation in multi-robot systems using Kmeans clustering and auction based mechanisms", Expert Systems With Applications, vol. 38, no. 6, (2011), pp. 6486-6491.

[5] P. R. Ferreira Jr, F. dos Santos and A. L. C. Bazzan, "WaskowRoboCup Rescue as multiagent task allocation among teams: experiments with task interdependencies", Auton Agent Multi-Agent Syst., vol. 20, (2010), pp. 421-443.

[6] Y. -T. Tian, M. Yang, X. -Y. Qi and Y. -M. Yang, "Multi-robot task allocation for fire-disaster response based on reinforcement learning", Conference of Machine Learning and Cybernetics, (2009) Baoding, China.

[7] L. Luo, N. Chakraborty and K. Sycara, "Multi-robot assignment algorithm for tasks with set precedence", Conference of Robotics and Automation, (2011) May 9-13, Shanghai, China.

[8] F. Hu, W. Xu and X. Li, "A modified particle swarm optimization algorithm for optimal allocation of earthquake emergency shelters", International Journal of Geographical Information Science, vol. 26, no. 9, (2012), pp. 1643-1666.

[9] L. Ying, Z. Wei, Z. Hongtao and C. Rong, "Research on dynamic emergency task allocation with MDP", Conference of Information Science and Engineering, (2010) December 4-6; Sponsore.

[10] E. G. Jones, M. b. Dias and A. Stentz, "Learning-enhanced Market-based Task Allocation for Disaster Response", tech. report CMU-RI-TR-06-48, Robotics Institute, Carnegie Mellon University, (2006).

[11] F. B. Luqman and M. L. Griss, "Overseer: A Mobile Context-Aware Collaboration and Task Management System for Disaster Response", Conference on Creating, Connecting and Collaborating through Computing, (2010), pp. 76-82.

[12] B. Lopez, S. Suarez and J. L. De La Rosa, "Task allocation in rescue operations using combinatorial auctions", Artificial Intelligence Research and Development, vol. 100, (2003), pp. 233-243.

[13] L. Dai, Z. Shen, T. Chen and Y. Chang, "Analysis and modeling of task scheduling in wireless sensor network based on divisible load theory", International Journal of Communication Systems, (2012).

[14] Z. C. Papazachos and H. D. Karatza, "Scheduling of frequently communicating tasks", International Journal of Communication Systems, (2012).

[15] F. Paterna, A. Acquaviva, A. Caprara, F. Papariello, G. Desoli and L. Benini, "Variability-Aware Task Allocation for Energy-Efficient Quality of Service Provisioning in Embedded Streaming Multimedia Applications", IEEE Transactions on Computers, vol. 61, no. 7, (2012), pp. 939-953.

[16] D. B. Shmoys and V. Tardos, "An approximation algorithm for the generalized assignment problem", Mathematical Programming, vol. 62, no. 3, (1993), pp. 461-474. 
[17] P. Scerri, A. Farinelli, S. Okamoto, et al., "Allocating tasks in extreme teams", Proceedings of the fourth international joint conference on autonomous agents and multiagent systems, (2005) July 25-29; Utrecht, Netherlands.

[18] M. Balmer, K. Meister, M. Rieser, et al., "Axhausen. Agent-based simulation of travel demand: Structure and computational performance of MATSim-T”, The 2nd TRB Conference on Innovations in Travel Modeling, (2008), Portland.

[19] M. Balmer, M. Rieser and K. Meister, "MATSim-T: Architecture and Simulation Times", Multi-Agent Systems for Traffic and Transportation Engineering, (2009).

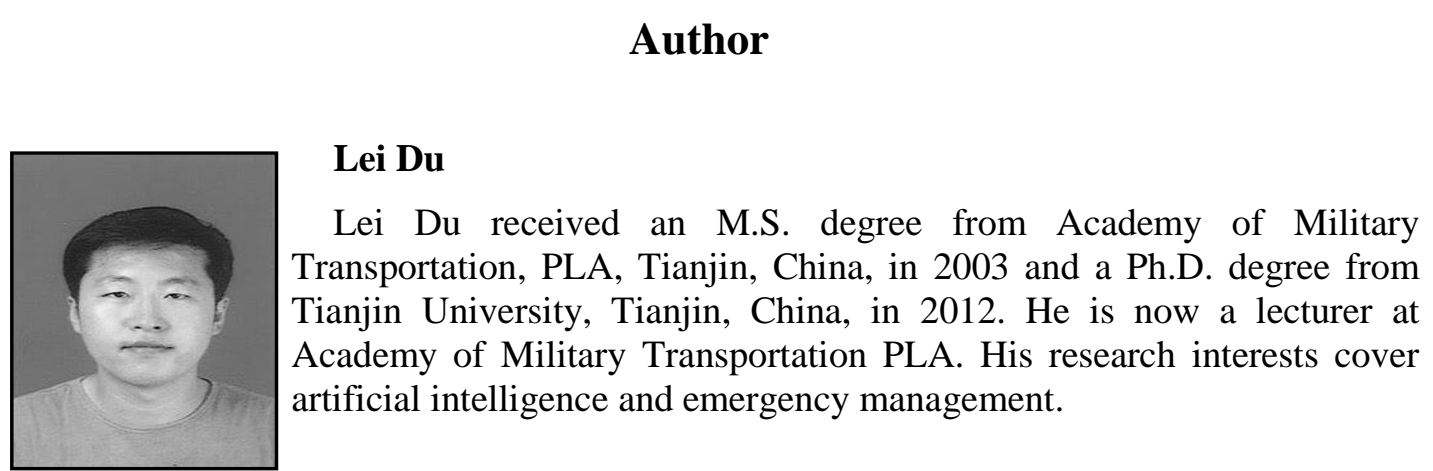

